\title{
Effect of chromosomal reorganizations on morphological covariation of the mouse mandible: insights from a Robertsonian system of Mus musculus domesticus
}

Jessica Martínez-Vargas ${ }^{1}$, Francesc Muñoz-Muñoz ${ }^{1 *}$, Nuria Medarde ${ }^{1}$, María José López-Fuster ${ }^{2}$ and Jacint Ventura ${ }^{1}$

\begin{abstract}
Introduction: Morphological integration and modularity depend on genetic covariation between traits, which emerges from pleiotropic effects of single loci and genetic linkage between loci. Since chromosomal reorganizations alter meiotic recombination, they might modify groups of linked genes and entail the fixation of new alleles with new pleiotropic effects. As a result, they could contribute to the intraspecific variation of the covariance structure of morphological traits. Although the mouse mandible has long been studied in terms of development and evolution, little is known about how its covariance structure varies in natural populations with chromosomal reorganizations. Consequently, here we analyzed the magnitude and patterns of morphological covariation of mandible shape in groups of mice with different karyotypes from a Robertsonian system of Mus musculus domesticus.
\end{abstract}

Results: The organization of the mouse mandible into two main modules was confirmed in all chromosomal groups, since $R V$ coefficients for the corresponding subdivision of landmarks were always significant. However, substantial variation in the magnitude of integration was detected between groups, especially when the effect of allometry was not removed. A significant positive correlation between differences in magnitude of integration of the symmetric component of shape and karyotypic distances between groups was detected when not correcting for size. Moreover, the degree of dependence of symmetric shape variation on size showed a negative association with the chromosome number and a positive association with the magnitude of integration. All groups showed similar patterns of morphological integration of the mandible, especially regarding the symmetric component of shape. However, the display of landmark displacements and the computation of vector angles highlighted some differences. In addition, distances between groups in terms of covariation matrices of the symmetric component were positively correlated with geographic distance.

Conclusions: Robertsonian translocations do not alter the organization of the mouse mandible into two main modules, but do affect the magnitude of integration between them. This effect is mainly due to changes in the allometric relationship. In the 'Barcelona' Robertsonian system, geographically structured sources of variation seem to affect the patterns of integration by producing parallel variation in separate developmental pathways. Overall, our results suggest that Robertsonian translocations could play a role in intraspecific differentiation processes by producing changes in the covariance structure of morphological traits.

Keywords: 'Barcelona' Robertsonian system, Mandible, Modularity, Morphological covariation, Morphological integration, Mus musculus domesticus, Robertsonian translocations

\footnotetext{
* Correspondence: francesc.munozm@uab.cat

'Departament de Biologia Animal, de Biologia Vegetal i d'Ecologia,

Universitat Autònoma de Barcelona, Facultat de Biociències, Bellaterra,

E-08193 Cerdanyola del Vallès, Spain

Full list of author information is available at the end of the article
} 


\section{Introduction}

Organisms are composed of several parts that need to be coordinated in order to allow them to function as a whole. In a morphological context, this coordination is known as morphological integration [1], and it is expressed through statistical covariation. However, not all parts within an organism covariate with each other to the same extent. This heterogeneity in the degree of covariation is the basis of the concept of modularity [2]. Accordingly, modules have been defined as complexes of tightly integrated traits, which are relatively independent from other such complexes [3]. Therefore, modularity and integration are complementary concepts that emphasize different aspects of covariation.

Morphological traits originate from the ensemble of molecular and cellular processes taking place during development, which are known as developmental pathways [4]. Consequently, covariation between morphological traits also arises during development, specifically from direct interactions between developmental pathways and parallel variation of separate developmental pathways. Covariation emerging from direct interactions originates in the pathways themselves through different mechanisms, such as the division of a precursor tissue into parts that respectively give rise to a different trait, or the transmission of variation through inductive signaling from one pathway to another. Instead, covariation emerging from parallel variation is due to the simultaneous influence of the same external factor (e.g. environmental conditions) on separate developmental pathways [4].

A substantial component of morphological covariation is genetic covariation, which can arise from genetic linkage between loci and pleiotropic effects of single loci [3]. Presumably, both sources of genetic covariation can be affected by chromosomal reorganizations such as Robertsonian $(\mathrm{Rb})$ translocations, which consist in the fusion of two non-homologous acrocentric chromosomes at their centromeres to originate a metacentric chromosome [5]. $\mathrm{Rb}$ translocations cause a decline in chiasma frequency and a more distal distribution of chiasmata during meiotic recombination [6-10], which could affect the linkage between alleles of loci that influence different traits within a structure. Besides, the reduction in recombination entailed by $\mathrm{Rb}$ translocations can prompt the fixation of different positively selected alleles [11], which may have different pleiotropic effects [12-15]. In relation to this, the hybridization between chromosomally different populations, differing in their fixed alleles, can entail developmental alterations and hence changes in terms of genetic covariation between traits in the subsequent generations [14].

The Western European house mouse (Mus musculus domesticus Schwarz and Schwarz, 1943 [16]) constitutes a model organism for the study of evolutionary processes linked to chromosomal reorganizations, as it shows great karyotypic diversity mainly due to $\mathrm{Rb}$ translocations [5]. Within the distributional area of Mus musculus domesticus, there are many geographic regions in which populations with different sets of metacentrics hybridize with each other and/or with populations with the standard (St) karyotype (40 acrocentric chromosomes). Jointly, these sets of populations are called $\mathrm{Rb}$ systems [5]. One of these, named 'Barcelona' $\mathrm{Rb}$ system, is present on the Northeastern Iberian Peninsula, specifically in part of the provinces of Barcelona, Tarragona and Lleida [17-20]. The set of metacentrics that characterizes this Rb system consists of $\mathrm{Rb}(3.8), \mathrm{Rb}(4.14), \mathrm{Rb}(5.15), \mathrm{Rb}(6.10), \mathrm{Rb}(7.17)$, $\mathrm{Rb}(9.11)$ and $\mathrm{Rb}(12.13)$, where the pairs of numbers refer to the acrocentric autosomes that gave rise to the metacentric in question. In this system, the frequency of metacentrics is distributed in a staggered way over $5,000 \mathrm{~km}^{2}$, leading to a progressive reduction in diploid number towards the center of the zone [20].

The mouse mandible has long been useful as a model system to study the development and evolution of complex morphological structures $[21,22]$. The mandible of the mouse has been divided into two functional modules: a distal one bearing the teeth (alveolar region), and a proximal one that articulates with the skull and constitutes the attachment point for most of the masticatory muscles (ascending ramus) [21]. The study of the genetic basis of the mandible shape has revealed that genetic modularity also occurs in this structure, in the same way as functional modularity does [23-25]. Besides, two concurring developmental modules can be distinguished in the mouse mandible, according to several lines of evidence $[26,27]$. Furthermore, evolutionary independence between these two mandibular modules has also been detected [28]. Instead, scarce analyses have been performed on the intraspecific variation of the covariance structure of the mouse mandible in natural populations. Similarly, there is a shortage of studies on how changes in the covariance structure prompted by karyotypic variation can contribute to the processes of morphological evolution (but see $[29,30])$. Covariance structure is a population-level feature whose role in dictating the directions and pace of evolutionary transformations has long been discussed [31,32]. In this regard, strong integration has been considered to constrain evolution because it implies that potentially favorable changes in some traits could entail adverse changes in associated traits [2]. Instead, modularity has been regarded as a driving force of evolution because it enables changes in certain traits to happen without affecting notably the rest of traits, thereby making it easier for evolutionary transformations to occur [2]. Keeping this in mind, it seems plausible that differences between populations in terms of their covariance structure could make them differ in their evolvability, or ability to evolve. If 
evolvability was itself the object of natural selection, which is actually a controversial hypothesis [33], variation between populations would then be expected to drive evolution within species. In this context, if chromosomal reorganizations turned out to affect the covariance structure, they could play a role in the evolvability of populations. In order to shed light on these topics, the present study aims to analyze the structure of morphological covariation of the mandible in wild populations of Mus musculus domesticus, and to assess the effect of $\mathrm{Rb}$ translocations on it.

Since the 'Barcelona' $\mathrm{Rb}$ system is characterized by a relatively large number of metacentrics and a wide range of diploid numbers, we considered it to be suitable for this study. While conducting it, several specific objectives were approached. In order to determine how $\mathrm{Rb}$ translocations affect morphological covariation of the mouse mandible, we assessed the magnitude and patterns of integration of this structure and tested its bimodular organization in groups with different karyotypes. Besides, given that allometry is considered to be a strong integrating factor [27], we evaluated its connection with these chromosomal reorganizations and its effect on the covariance structure of the mandible. As stated above, morphological covariation can result from both direct interactions between developmental pathways and parallel variation of separate developmental pathways. While covariation between symmetric shape changes can result from both sources, covariation between asymmetric shape changes is only due to direct developmental interactions [4]. In order to know the relative importance of direct interactions and parallel variation of developmental pathways in generating morphological covariation in the mouse mandible, we studied symmetric and asymmetric shape changes separately, which are usually termed symmetric component and asymmetric component of shape variation respectively. Lastly, because chromosomal variation in the 'Barcelona' $\mathrm{Rb}$ system is geographically arranged [18,20], we also assessed the effect of geographic distance on the covariance structure of the mandible.

\section{Results}

\section{Sources of shape and size variation}

Procrustes analyses of variance (ANOVAs) carried out on the replicated subsample revealed a significant effect of the individual and side factors, as well as their interaction, on mandible shape (Table 1). On the other hand, a significant effect of the individual factor and the interaction term, but not of the side factor, was detected on mandible size (Table 2). Since the significant effect of the interaction term indicates that variation in fluctuating asymmetry exceeds variation resulting from measurement error, subsequent analyses were based on a single digitization of landmarks per hemimandible.
Table 1 Procrustes ANOVA conducted on the replicated subsample to evaluate the influence of measurement error on shape data

\begin{tabular}{llllll}
\hline \multirow{2}{*}{ Effect } & \multicolumn{2}{l}{ Shape } & & & \\
\cline { 2 - 6 } & SS & df & MS & $\boldsymbol{F}$ & $\boldsymbol{P}$ \\
\hline Individual & 1.919 & 4520 & $4.245 \times 10^{-4}$ & 6.72 & $<0.001$ \\
Side & 0.024 & 20 & $1.173 \times 10^{-3}$ & 18.56 & $<0.001$ \\
Individual $\times$ Side & 0.286 & 4520 & $6.318 \times 10^{-5}$ & 4.62 & $<0.001$ \\
Measurement error & 0.248 & 18160 & $1.367 \times 10^{-5}$ & & \\
\hline
\end{tabular}

SS, sum of squares; df, degrees of freedom; MS, mean squares; $F, F$ statistic $P, P$-value.

Procrustes ANOVAs conducted on each chromosomal group separately (40St, 40Rb, Rb(38-39), Rb(34-37), Rb (31-33), $\mathrm{Rb}(27-30))$ consistently showed significant differences between individuals, regarding both shape and size variation. While directional asymmetry in shape was detected in all groups, directional asymmetry in size was only detected in group $\mathrm{Rb}(31-33)$ (see Additional file 1).

\section{Allometry}

A significant dependence of the symmetric component of shape on size was detected in all groups $(P<0.001)$. Size accounted for low-to-moderate fractions of this component (40St: 10.78\%; 40Rb: 8.30\%; Rb(38-39): 12.88\%; Rb (34-37): 13.07\%; Rb(31-33): 13.63\%; $\mathrm{Rb}(27-30): 23.78 \%)$. The asymmetric component of shape variation showed a significant dependence on size asymmetry $(P<0.05)$ only in groups $40 \mathrm{St}, 40 \mathrm{Rb}$ and $\mathrm{Rb}(27-30)$. On the whole, fairly low percentages of this component were predicted by size (40St: $2.41 \%$; 40Rb: 4.08\%; $\mathrm{Rb}(38-39): 1.94 \%$; $\mathrm{Rb}$ (34-37): 1.76\%; $\mathrm{Rb}(31-33): 1.08 \%$; $\mathrm{Rb}(27-30): 3.56 \%)$. The linear regression revealed a significant negative association between the percentage of symmetric shape variation explained by size and chromosome number $(r=-0.86$, $\mathrm{p}<0.05)$. No significant association was observed for the asymmetric component $(\mathrm{r}=0.15, \mathrm{p}=0.78)$.

\section{Modularity and magnitude of integration}

The $R V$ coefficient for the tested partition of landmarks was significant for both components of shape variation in all groups, both for raw and size-corrected data

Table 2 Two-way ANOVA conducted on the replicated subsample to evaluate the influence of measurement error on size data

\begin{tabular}{llllll}
\hline \multirow{2}{*}{ Effect } & \multicolumn{6}{l}{ Centroid size } & & \\
\cline { 2 - 6 } & SS & df & MS & $\boldsymbol{F}$ & $\boldsymbol{P}$ \\
\hline Individual & 8.068 & 226 & $3.570 \times 10^{-2}$ & 191.33 & $<0.001$ \\
Side & 0.001 & 1 & $4.670 \times 10^{-4}$ & 2.51 & 0.115 \\
Individual $\times$ Side & 0.042 & 226 & $1.870 \times 10^{-4}$ & 2.28 & $<0.001$ \\
Measurement error & 0.074 & 908 & $8.200 \times 10^{-5}$ & & \\
\hline
\end{tabular}

SS, sum of squares; df, degrees of freedom; MS, mean squares; $F, F$ statistic; $P, P$-value. 
(Figure 1). Therefore, the predicted hypothesis of modularity was always confirmed. Overall, the $R V$ coefficients corresponding to the symmetric component were higher than those corresponding to the asymmetric component (Figure 1 and Table 3). When using the rarefaction procedure to standardize the $R V$ coefficients to a given sample
A Symmetric Component
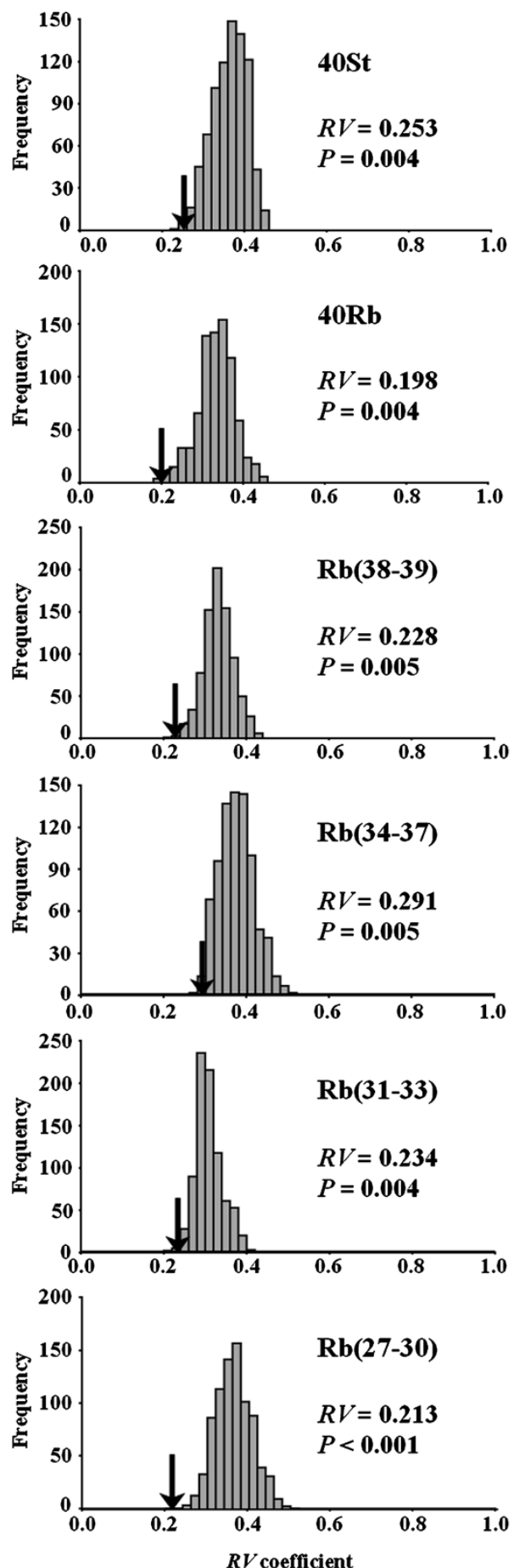

B Asymmetric Component
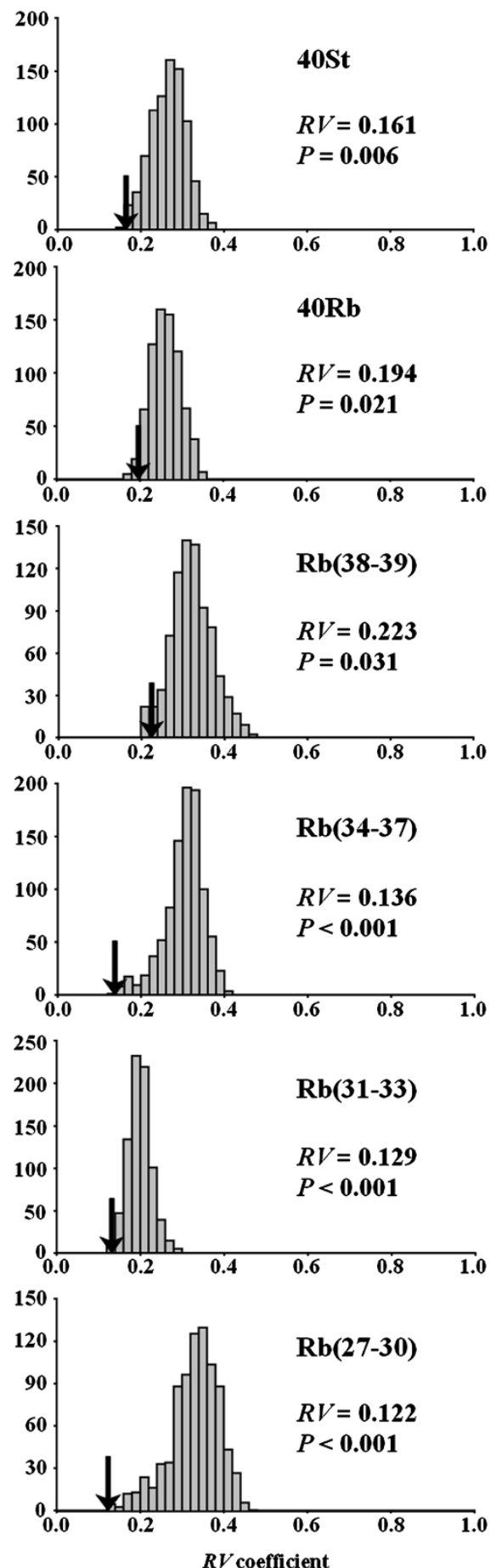

Figure 1 Distributions of $R V$ coefficients for the symmetric (A) and asymmetric (B) components for size-corrected data. The values of $R V$ coefficients for the partition of landmarks concurrent with the hypothesis of modularity are indicated by arrows and highlighted. $P$-values $(P)$ are adjusted through the FDR procedure. 
Table $3 R V$ coefficients non-standardized (NS) and standardized (S) to the same sample size

\begin{tabular}{llllll}
\hline Group & \multicolumn{2}{l}{ Symmetric component } & & \multicolumn{2}{l}{ Asymmetric component } \\
\cline { 2 - 3 } & NS & S & & NS & S \\
\hline 40St & $0.253(0.357)$ & 0.402 & & $0.161(0.164)$ & 0.341 \\
40Rb & $0.198(0.300)$ & 0.344 & & $0.194(0.204)$ & 0.431 \\
Rb(38-39) & $0.228(0.394)$ & 0.416 & & $0.223(0.227)$ & 0.473 \\
Rb(34-37) & $0.291(0.448)$ & 0.467 & & $0.136(0.132)$ & 0.389 \\
$\mathbf{R b}(31-33)$ & $0.234(0.372)$ & 0.406 & & $0.129(0.127)$ & 0.359 \\
$\mathbf{R b}(\mathbf{2 7 - 3 0 )}$ & $0.213(0.530)$ & 0.378 & & $0.122(0.117)$ & 0.302
\end{tabular}

Values between parentheses stand for $R V$ s obtained from raw data.

size $(n=77)$, they increased for both shape components. However, in both cases the pattern across groups was consistent with that displayed by the non-standardized $R V$ coefficients (Table 3 ).

The linear regression revealed a significant positive dependence of the magnitude of integration of the symmetric component on allometric percentage $(r=0.92, P<0.01)$.

Mantel and partial Mantel tests detected a significant positive correlation between distances in magnitude of integration of the symmetric component, calculated from raw data, and karyotypic, but not geographic, distances between groups (Table 4). This association was not obtained with size-corrected data. As for the asymmetric component, analyses with both raw and size-corrected data revealed no significant association between differences in the magnitude of integration and karyotypic or geographic distances.

\section{Patterns of integration}

According to the results of the principal component (PC) analyses performed on size-corrected data, mandibular shape variation was mainly concentrated in the first few PCs. In all chromosomal groups, the first two PCs jointly accounted for a substantial fraction of shape variation, for both the symmetric and asymmetric components (Tables 5 and 6). Besides, all groups showed a similar distribution of the percentages of total variance accounted for by the first ten PCs (Tables 5 and 6). When the shape changes associated with the first two PCs were displayed as diagrams of eigenvectors, most of the variation was concentrated in the ascending ramus, particularly in the coronoid and condylar processes (Figure 2). While the pattern of variation corresponding to PC1 of the symmetric component turned out to be quite similar between groups, conspicuous differences affecting the angular and condylar processes were detected in group 40St (Figure 2A); in fact, vector angles between this group and the others were by far the greatest (Table 7). Regarding the pattern of shape changes associated with $\mathrm{PC} 2$ of the symmetric component, noticeable differences were detected between groups (Figure $2 \mathrm{~A})$. In this case, groups $40 \mathrm{St}$ and $\mathrm{Rb}(27-$ 30) showed the comparatively most distinct patterns of shape variation (Table 8). As for both PC1 and PC2 of the asymmetric component, some differences in the displacement direction of landmarks were detected between groups (Figure 2B), which was supported by vector angles (Tables 7 and 8).

Significant positive correlations were detected when comparing the covariance matrices of both the symmetric and asymmetric components between groups (Table 9). Correlations between the covariance matrices of both components of each group were also positive and significant, and the correlation coefficient increased as the mean diploid number of the group decreased (Table 9).

According to both Mantel and partial Mantel tests, distances in patterns of integration of the symmetric component, calculated from both raw and size-corrected data, were positively and significantly correlated with geographic distances between groups (Table 4). Although the Mantel test also detected a significant positive correlation between distances in patterns of integration of the symmetric component, computed from size-corrected data, and karyotypic distances, this association was not detected by the partial Mantel test (Table 4). Distances in

Table 4 Mantel and partial Mantel tests correlations between distances in magnitude (RV) and patterns (1-r) of morphological integration, and karyotypic and geographic distances

\begin{tabular}{|c|c|c|c|c|c|}
\hline & & \multicolumn{2}{|c|}{ Geographic distance } & \multicolumn{2}{|c|}{ Karyotypic distance } \\
\hline & & Mantel & Partial Mantel & Mantel & Partial Mantel \\
\hline \multirow[t]{4}{*}{ Raw data } & $R V_{\text {sym }}$ & 0.01 & -0.49 & $0.60^{*}$ & $0.72^{*}$ \\
\hline & $R V_{\text {asym }}$ & 0.17 & 0.00 & 0.32 & 0.27 \\
\hline & $1-r_{\text {sym }}$ & $0.75^{*}$ & $0.69^{*}$ & 0.39 & -0.03 \\
\hline & $1-r_{\text {asym }}$ & -0.02 & 0.14 & -0.25 & -0.28 \\
\hline \multirow[t]{4}{*}{ Size-corrected data } & $R V_{\text {sym }}$ & 0.00 & 0.23 & -0.32 & -0.39 \\
\hline & $R V_{\text {asym }}$ & 0.11 & -0.04 & 0.26 & 0.24 \\
\hline & $1-r_{\text {sym }}$ & $0.77^{*}$ & $0.68^{*}$ & $0.50^{*}$ & 0.14 \\
\hline & $1-r_{\text {asym }}$ & -0.06 & 0.12 & -0.31 & -0.29 \\
\hline
\end{tabular}


Table 5 Percentages of total variance accounted for by the first ten PCs of the symmetric component

\begin{tabular}{lllllllllll}
\hline & PC1 & PC2 & PC3 & PC4 & PC5 & PC6 & PC7 & PC8 & PC9 & PC10 \\
\hline 40St & 18.30 & 13.52 & 11.15 & 10.15 & 6.62 & 5.96 & 4.82 & 3.99 & 3.27 & 2.82 \\
40Rb & 17.89 & 16.45 & 9.22 & 8.42 & 7.68 & 5.43 & 4.90 & 4.46 & 3.78 & 3.40 \\
$\mathbf{R b}(\mathbf{3 8 - 3 9 )}$ & 17.64 & 13.07 & 9.22 & 8.40 & 7.69 & 6.92 & 5.97 & 4.30 & 4.17 & 3.46 \\
$\mathbf{R b}(\mathbf{3 4 - 3 7 )}$ & 20.58 & 12.85 & 9.52 & 9.02 & 6.35 & 5.85 & 5.37 & 4.79 & 3.55 & 2.97 \\
$\mathbf{R b}(\mathbf{3 1 - 3 3 )}$ & 19.79 & 11.03 & 10.63 & 8.23 & 7.17 & 6.07 & 5.35 & 4.37 & 3.53 & 3.17 \\
$\mathbf{R b}(\mathbf{2 7 - 3 0})$ & 19.82 & 11.53 & 10.84 & 9.39 & 7.20 & 6.42 & 4.94 & 4.33 & 3.57 & 3.23 \\
\hline
\end{tabular}

the patterns of integration of the asymmetric component were not correlated with karyotypic nor geographic distances, either with raw or size-corrected data (Table 4).

\section{Discussion}

$\mathrm{Rb}$ translocations affect the magnitude of integration but not modularity

The results of the tests of modularity lent support to the notion that the mouse mandible consists of two primary modules, the alveolar region and the ascending ramus [25-28]. Besides, the existence of the proximal module is backed by the fact that shape changes within that region were comparatively more coordinated, and that most of the variation in mandible shape was particularly concentrated there $[24,34,35]$. Despite the confirmation of such modular organization, the fact that only a few PCs accounted for a large part of shape variation indicates that the mouse mandible has a certain degree of overall integration [27,36-39]. As proved, the modular configuration of the mouse mandible is not altered by $\mathrm{Rb}$ translocations, regardless of the number of metacentrics. However, the magnitude of integration between the two mandibular modules varied notably among chromosomal groups, especially when allometry was not removed. Because the effects of size simultaneously affect all the parts of a structure, allometry is usually considered to have an integrating influence and thus to obscure modular organizations [27]. In our study, despite significant allometric relationships, modularity was always detected. However, and as expected, magnitudes of integration between the two modules were higher when not correcting for the effect of size. Moreover, magnitudes of integration of the symmetric component of shape increased along with the percentage of allometry, whereas this percentage was negatively associated with diploid number. Thus, the greater dependence of shape on size in groups with low diploid numbers seems to be the reason why they showed a greater magnitude of integration between the two mandibular modules. At the same time, this would explain why Mantel tests performed with raw data detected an association between differences in karyotype and differences in magnitude of integration between groups. Previous studies have found many quantitative trait loci (QTLs) affecting the size and shape of the mandible [40]. Since $\mathrm{Rb}$ translocations reduce meiotic recombination [7], their accumulation could progressively increase the probability of linkage between QTLs affecting size and QTLs affecting shape, which would explain the greater association between size and shape as diploid number decreases.

When the effect of allometry was removed, no linear association was detected between differences in magnitude of integration of the mandible and neither geographic nor karyotypic distances, for none of the two components of shape variation. However, these results do not necessarily preclude an effect of $\mathrm{Rb}$ translocations on the integration of this structure. As previously stated (see Introduction), these chromosomal reorganizations can affect morphological covariation by modifying the linkage between alleles of loci influencing different mandibular traits, and by entailing the fixation of positively selected alleles. The progressively lower $R V$ coefficients obtained in groups $\mathrm{Rb}(34-37), \mathrm{Rb}$ (31-33) and $\mathrm{Rb}(27-30)$ could be due to the modification of the linkage between alleles. Given that metacentrics show the lowest chiasma frequency around the centromere [9], the formation of $\mathrm{Rb}$ translocations could progressively imply the emergence of new linkage groups around the centromere of the newly-formed metacentrics. In connection with this, far more QTLs have been assigned to

Table 6 Percentages of total variance accounted for by the first ten PCs of the asymmetric component

\begin{tabular}{lllllllllll}
\hline & PC1 & PC2 & PC3 & PC4 & PC5 & PC6 & PC7 & PC8 & PC9 & PC10 \\
\hline 40St & 18.30 & 11.90 & 9.87 & 8.94 & 7.99 & 7.01 & 5.51 & 4.72 & 3.90 & 3.22 \\
40Rb & 15.30 & 12.74 & 11.41 & 9.52 & 6.94 & 6.42 & 5.82 & 4.58 & 4.20 & 3.68 \\
$\mathbf{R b}(38-39)$ & 20.28 & 16.17 & 11.25 & 7.16 & 6.20 & 5.35 & 5.13 & 4.29 & 3.41 & 2.73 \\
$\mathbf{R b}(\mathbf{3 4 - 3 7 )}$ & 20.78 & 14.01 & 10.63 & 8.63 & 6.52 & 5.14 & 4.72 & 4.53 & 3.50 & 2.80 \\
$\mathbf{R b}(\mathbf{3 1 - 3 3 )}$ & 12.60 & 12.01 & 11.24 & 8.34 & 6.91 & 6.51 & 6.17 & 5.16 & 4.49 & 3.87 \\
$\mathbf{R b}(\mathbf{2 7 - 3 0})$ & 22.32 & 15.22 & 10.55 & 8.46 & 6.72 & 4.96 & 4.64 & 3.76 & 3.35 & 2.73 \\
\hline
\end{tabular}




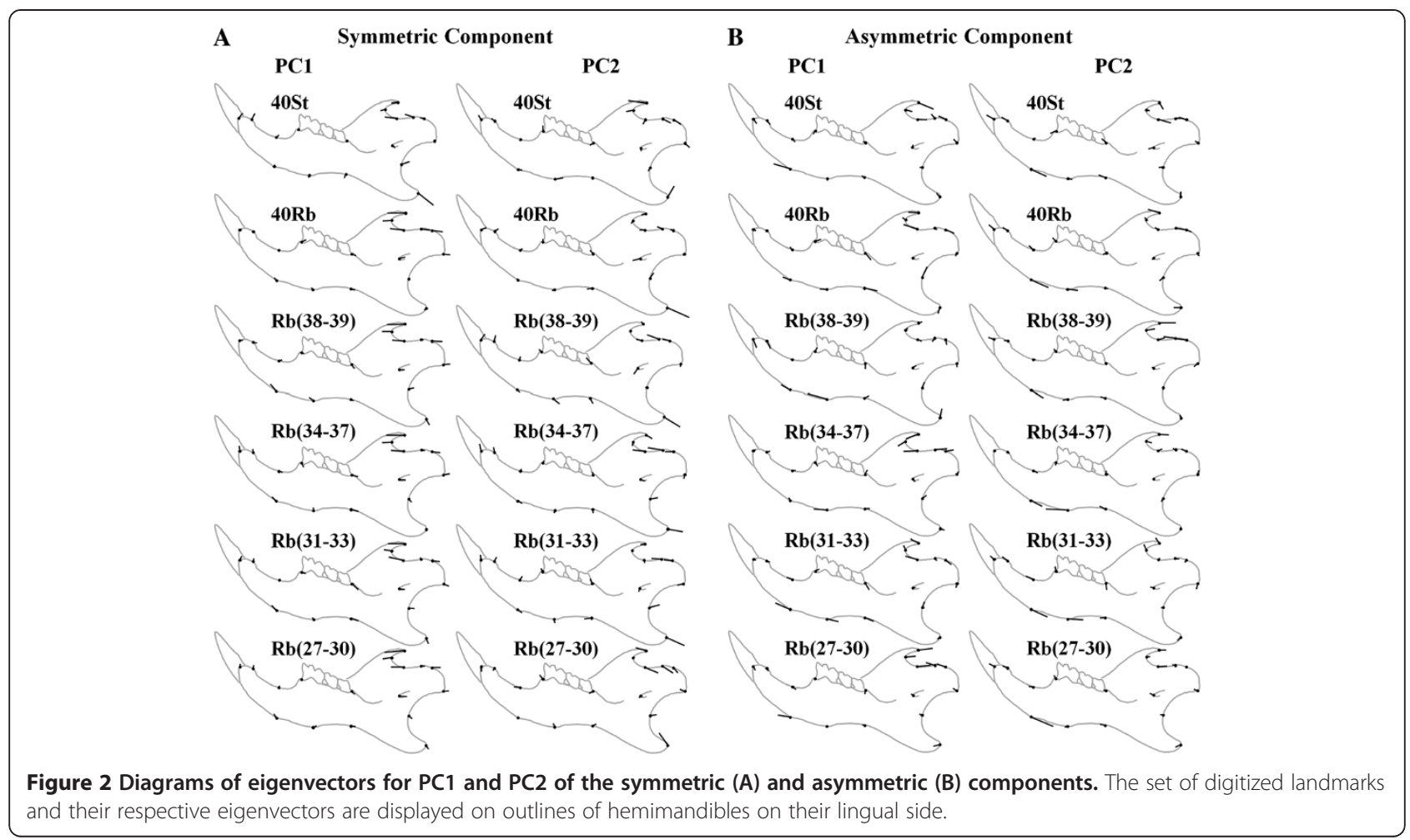

the ascending ramus than to the alveolar region of the mouse mandible, and several of them are located close to the centromeres of acrocentric chromosomes [23]. Therefore, it is likely that the new linkage groups prompted by $\mathrm{Rb}$ translocations would include several QTLs with an effect only on the ascending ramus. If that was the case, covariation, and so integration, within this module would increase along with the amount of $\mathrm{Rb}$ translocations, and this would explain the observed decrease in the magnitude of integration of the mandible, in terms of $R V$, along with the decrease in diploid number. Instead, the increase in the magnitude of integration of the asymmetric component detected in group $\mathrm{Rb}(38-39)$ with respect to St populations might not be explained by this mechanism, but by the higher rate of fixation of alleles in metacentrics due to the decrease in meiotic recombination

Table 7 Vector angles in degrees for PC1

\begin{tabular}{|c|c|c|c|c|c|c|}
\hline & $40 \mathrm{St}$ & $40 \mathrm{Rb}$ & $\begin{array}{l}\mathrm{Rb} \\
(38-39) \\
\end{array}$ & $\begin{array}{l}\mathrm{Rb} \\
(34-37)\end{array}$ & $\begin{array}{l}\text { Rb } \\
(31-33)\end{array}$ & $\begin{array}{l}\mathrm{Rb} \\
(27-30)\end{array}$ \\
\hline 40St & 71.29 & $68.32^{*}$ & 71.68 & $65.49^{*}$ & 83.50 & $29.99 * *$ \\
\hline 40Rb & 76.14 & $48.23^{* *}$ & 73.06 & $52.06^{* *}$ & $43.97^{* *}$ & $67.05^{*}$ \\
\hline $\mathrm{Rb}(38-39)$ & $60.87^{*}$ & $26.71^{* *}$ & 84.21 & 81.65 & 87.77 & 75.24 \\
\hline $\mathrm{Rb}(34-37)$ & $67.80^{*}$ & $24.29 * *$ & $25.80^{* *}$ & $44.93^{* *}$ & 78.75 & $47.47^{* *}$ \\
\hline $\mathrm{Rb}(31-33)$ & $57.73^{*}$ & $29.90^{* *}$ & $22.16^{* *}$ & $20.83^{* *}$ & $50.01^{* *}$ & 80.46 \\
\hline $\mathrm{Rb}(27-30)$ & $63.36^{*}$ & $22.95^{* *}$ & $23.58^{* *}$ & $16.93^{* *}$ & $22.64^{* *}$ & $43.57^{* *}$ \\
\hline
\end{tabular}

Values between PC1 of symmetric and asymmetric components of each group (on the diagonal) and between PC1s of the symmetric (below) and the asymmetric (above) components among groups. ${ }^{*} P<0.05$; ${ }^{* *} P<0.001$. that they undergo [10,11]. In these metacentrics, the fixed alleles could affect covariation in two different ways. On the one hand, as pleiotropic effects vary together with alleles [12-15], the fixation of new favorable alleles in metacentrics would likely alter genetic covariation of morphological structures. On the other hand, it has been proved that hybrids between populations with different fixed alleles show greater genetic covariation than the parental generations [14]. In particular, when Renaud and collaborators [35] analyzed the strength of covariation between the two main mandibular modules in two subspecies of house mouse and the resulting hybrids, they found that it was higher in the filial 1 (F1) hybrids than in the parental groups and the filial 2 (F2) hybrids. Linking it to our study, given that new alleles are expected to become fixed in $\mathrm{Rb}$ translocations, hybridization between $\mathrm{St}$ and $\mathrm{Rb}$ mouse populations could

Table 8 Vector angles in degrees for PC2

\begin{tabular}{lllllll}
\hline & 40St & 40Rb & $\begin{array}{l}\mathbf{R b} \\
\mathbf{( 3 8 - 3 9 )}\end{array}$ & $\begin{array}{l}\mathbf{R b} \\
\mathbf{( 3 4 - 3 7 )}\end{array}$ & $\begin{array}{l}\mathbf{R b} \\
\mathbf{( 3 1 - 3 3 )}\end{array}$ & $\begin{array}{l}\mathbf{R b} \\
\mathbf{( 2 7 - 3 0 )}\end{array}$ \\
\hline 40St & 86.74 & $67.70^{*}$ & $56.84^{*}$ & 69.59 & $62.69^{*}$ & $42.51^{* *}$ \\
$40 \mathrm{Rb}$ & 88.22 & 73.35 & 85.20 & 76.60 & $34.91^{* *}$ & $62.15^{*}$ \\
$\mathrm{Rb}(38-39)$ & 88.23 & $42.30^{* *}$ & 82.22 & $49.28^{* *}$ & 79.73 & $43.81^{* *}$ \\
$\mathrm{Rb}(34-37)$ & 80.38 & $53.34^{* *}$ & $42.60^{* *}$ & 83.06 & 75.51 & $57.72^{*}$ \\
$\mathrm{Rb}(31-33)$ & 87.91 & $37.01^{* *}$ & $39.86^{* *}$ & $44.39^{* *}$ & 84.44 & $54.20^{* *}$ \\
$\mathrm{Rb}(27-30)$ & $64.34^{*}$ & $63.13^{*}$ & $55.74^{* *}$ & $49.40^{* *}$ & 70.47 & 85.71 \\
\hline
\end{tabular}

Values between PC2 of symmetric and asymmetric components of each group (on the diagonal) and between PC2s of the symmetric (below) and the asymmetric (above) components among groups. ${ }^{*} P<0.05 ;{ }^{* *} P<0.001$. 
Table 9 Correlation coefficients ( $r$ ) between covariance matrices

\begin{tabular}{lllllll}
\hline & 40St & 40Rb & $\begin{array}{l}\mathbf{R b} \\
(\mathbf{3 8 - 3 9 )}\end{array}$ & $\begin{array}{l}\mathbf{R b} \\
\mathbf{( 3 4 - 3 7 )}\end{array}$ & $\begin{array}{l}\mathbf{R b} \\
(\mathbf{3 1 - 3 3 )}\end{array}$ & $\begin{array}{l}\mathbf{R b} \\
\mathbf{( 2 7 - 3 0 )}\end{array}$ \\
\hline 40St & 0.473 & 0.587 & 0.432 & 0.414 & 0.626 & 0.644 \\
$\mathbf{4 0 R b}$ & 0.681 & 0.504 & 0.475 & 0.574 & 0.704 & 0.586 \\
$\mathbf{R b}(\mathbf{3 8 - 3 9 )}$ & 0.665 & 0.742 & 0.506 & 0.489 & 0.538 & 0.474 \\
$\mathbf{R b}(\mathbf{3 4 - 3 7 )}$ & 0.640 & 0.702 & 0.731 & 0.539 & 0.623 & 0.657 \\
$\mathbf{R b}(\mathbf{3 1 - 3 3 )}$ & 0.681 & 0.719 & 0.760 & 0.824 & 0.520 & 0.667 \\
$\mathbf{R b}(\mathbf{2 7 - 3 0 )}$ & 0.617 & 0.683 & 0.689 & 0.756 & 0.780 & 0.583 \\
\hline
\end{tabular}

Values between covariance matrices of symmetric and asymmetric components of each group (on the diagonal), and of symmetric (below) and asymmetric (above) components among groups. All coefficients are significant $(P<0.001)$.

probably give rise to hybrid populations showing greater covariation between the two mandibular modules. Bearing in mind the preceding argumentation, we suggest that the differences detected between chromosomal groups in terms of magnitude of integration of the asymmetric component might result from the balance between the divergent effects that the modification of genetic linkage, on the one side, and the differential fixation of alleles, on the other side, may have on morphological covariation in each of them. As it can be noticed by looking back on the results, this argumentation does not fit with the differences between groups in terms of the magnitude of integration of the symmetric component. As stated above, this component of shape variation, unlike the asymmetric one, takes account of variation due to the effect of external stimulus on developmental pathways, which can lead to covariation between traits if those factors affect different pathways simultaneously [4]. Therefore, the impact of certain external sources of variation could be responsible for that discrepancy between results regarding the symmetric and asymmetric components.

\section{Morphological covariation arises from different developmental sources}

The degree of congruence between symmetric and asymmetric covariation is said to provide evidence for the relative importance of the two developmental origins of morphological covariation, namely direct interactions between developmental pathways and parallel variation of separate developmental pathways $[4,30,34]$. When comparing the covariance matrices of the symmetric and asymmetric components of shape variation in each group, intermediate correlation coefficients were generally obtained, which agrees with the results obtained in previous studies [34]. This indicates that, although covariation between symmetric shape changes of the mandible arises from direct developmental interactions to some extent, a considerable amount of this covariation is actually due to the parallel variation of separate developmental pathways. Moreover, the fact that these correlation values increase as diploid number decreases suggests that the accumulation of new $\mathrm{Rb}$ translocations might entail a greater importance of direct interactions over parallel variation in generating morphological covariation in the mouse mandible. However, the mechanisms by which this may happen remain unknown.

\section{Variation in the patterns of integration is geographically structured}

Correlation coefficients between the covariance matrices of the chromosomal groups revealed that, in general, they share similar patterns of integration of the mandible. However, diagrams of landmark displacements and vector angles indicated that particular aspects of integration patterns differ between groups. The positive association detected between the differences in the patterns of integration of the symmetric component and the geographic distances between groups suggests that morphological covariation of the mandible is geographically structured. As mentioned above, symmetric covariation takes account of covariation between morphological traits that arises from the simultaneous effect of an external factor on separate developmental pathways. Among these factors, one can distinguish environmental conditions and allelic variability in genes involved in different developmental processes [4], which are sources of variation that can be geographically structured. The positive association detected between differences in the patterns of integration of the symmetric component and geographic distances between groups suggests that such patterns might be influenced by environmental factors and/or genetic differences due to isolation by distance. Several studies state that covariation patterns are remarkably similar between closely related species, as well as between groups belonging to the same species $[30,31,41,42]$, whereas others show that they can vary significantly at small taxonomic scales $[43,44]$. In reference to this, our results suggest that even though different populations of the same species may show, at a glance, considerably similar patterns of morphological covariation, intraspecific variability can still exist and be detectable. Besides, they highlight that the geographic structure of populations can affect their patterns of morphological integration, and that this effect seems to take place mainly through the simultaneous influence of geographically-structured external factors on separate developmental pathways involved in generating the morphological structure in question.

\section{Conclusions}

In the light of our results, we conclude that the organization of the mouse mandible into two modules is a stable attribute that is neither distorted by the presence of $\mathrm{Rb}$ translocations nor by the integrative effect of allometry. However, both the accumulation of $\mathrm{Rb}$ translocations 
and allometric relationships affect the magnitude of integration between modules. Notably, the magnitude of integration increases along with the number of $\mathrm{Rb}$ translocations due to a parallel increase in the amount of shape variation depending on size. Analyses of sizecorrected data also suggest that $\mathrm{Rb}$ translocations can affect the magnitude of integration, presumably by linking genes with different effects on mandible morphology and by giving rise to new combinations of alleles. In the first case, because of the genetic constitution of the mouse mandible, a decrease in the magnitude of integration is expected as the number of translocations rises due to an increase in covariation within the ascending ramus. In the second case, a greater magnitude of integration is expected in populations with a mixture of different karyotypes.

Notwithstanding the fact that populations with different sets of $\mathrm{Rb}$ translocations share similar patterns of covariation, they actually differ in particular aspects. This variation follows a geographic structure, probably because external sources of variation that affect separate developmental pathways in parallel, such as environmental factors or genetic differences, are geographically structured.

Overall, our study proves that the covariance structure of the mouse mandible shows intraspecific variation between natural populations, not only due to the presence of $\mathrm{Rb}$ translocations, but also because of the effect of geographically structured factors. Chromosomal reorganizations are usually thought to take part in speciation processes by acting as barriers to gene flow [11]. Our results support the notion that chromosomal reorganizations can also modify the patterns of morphological integration. Thus, chromosomal reorganizations could also play a role in differentiation processes by producing changes in the covariance structure of morphological traits that might entail evolution of populations in divergent directions.

\section{Materials and methods}

\section{Sample and data acquisition}

The sample consisted of 1233 right and left hemimandibles from 619 adult wild mice (308 females and 311 males) from the 'Barcelona' $\mathrm{Rb}$ system and surrounding St populations. Chromosome preparations were obtained directly from bone marrow [45] and stained using Wright's stain for G-banding [46].

Once their karyotypes were determined (see Additional file 2), the specimens were classified into six groups on the basis of their diploid number: 40St, $\mathrm{n}=86$; $40 \mathrm{Rb}, \mathrm{n}=77 ; \mathrm{Rb}(38-39), \mathrm{n}=84 ; \mathrm{Rb}(34-37), \mathrm{n}=107$; $\mathrm{Rb}(31-33), \mathrm{n}=159 ; \mathrm{Rb}(27-30), \mathrm{n}=106$. Group 40St included specimens with the standard karyotype (40 acrocentric chromosomes) from localities where there is no evidence of metacentrics. $40 \mathrm{Rb}$ stands for the group including individuals with the standard karyotype from localities where $\mathrm{Rb}$ translocations have been reported. Hemimandibles left and right of each individual were detached and laid on a black cardboard. Images of their lingual view, together with a scale in millimeters, were obtained with a Nikon COOLPIX P90 digital camera placed $21.5 \mathrm{~cm}$ from the cardboard. Seventeen twodimensional landmarks were digitized in all the images using the tpsDig2 software (Figure 3A and Additional file 3). In a subsample of 454 hemimandibles (277 right and 277 left; $36.82 \%$ of the total), a set of landmarks was digitized three times.

\section{Analyses of integration and modularity}

Morphological integration and modularity of the mandible were analyzed by implementing the geometric morphometric methods included in the MorphoJ software, version 1.05e [47]. Size was estimated through centroid size [48]. The landmark configurations of all left hemimandibles were reflected to their mirror images. Then, the configurations obtained via reflection and those of the right hemimandibles were superimposed through a generalized least-squares Procrustes fit and were projected onto the shape tangent space $[34,48,49]$. As a result of this procedure, variation due to size, position and orientation was eliminated, and so shape information was extracted $[24,48]$.

Coordinates resulting from the Procrustes fit (Procrustes coordinates) were then analyzed by means of multivariate statistics methods. First of all, Procrustes ANOVAs were conducted on the three sets of replicated configurations in order to assess the influence of measurement error on size and shape data [50,51]. Individual and side were entered as random and fixed factors respectively, and Procrustes distances as the dependent variable. The individual factor stands for individual variation, the side factor for directional asymmetry, and the interaction between these two factors represents fluctuating asymmetry $[47,49,52]$. Measurement error was quantified from the residual variance component between replicates [50]. Since size and shape variation due to measurement error was significantly lower than variation in fluctuating asymmetry, the ensuing analyses (listed below) were based on a single digitization of landmarks per hemimandible. Given that the study was intended to assess the effect of different numbers of $\mathrm{Rb}$ translocations on the patterns of morphological integration and modularity, these analyses were conducted for each chromosomal group separately.

To begin with, Procrustes ANOVAs for size and shape were carried out. These analyses separated total variation into its symmetric component, which is the variation between individuals in terms of the averages of the original and reflected landmark configurations, and its asymmetric component, which is the variation within individuals 


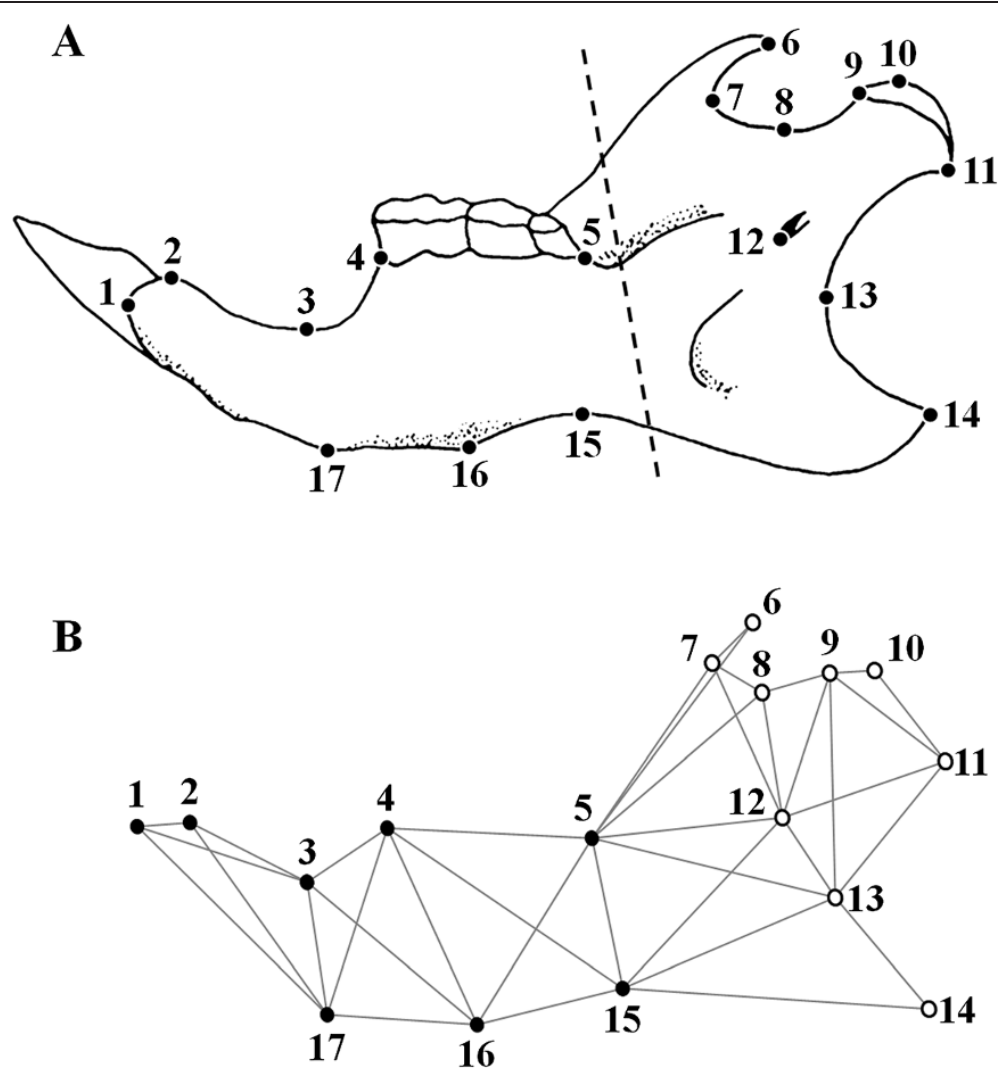

Figure 3 Digitized landmarks and adjacency graph. A) Layout of the landmarks on the lingual view of right hemimandible. The dashed line divides the set of landmarks into two subsets concurrent with the two functional modules. B) Adjacency graph defining spatially contiguous partitions of landmarks.

regarding the landmark deviations of the reflected configuration from the original one [30,34,51].

Allometry, that is, the scaling relationship between shape and size, was then evaluated through multivariate regressions of both the symmetric and asymmetric components of shape variation onto symmetric and asymmetric centroid size respectively. Significations were obtained through permutation tests with 10,000 iterations [53,54]. The association between chromosome number and percentage of shape variation explained by size was tested through the regression of the allometric percentages of groups onto their mean diploid number, for both the symmetric and asymmetric components. Since a significant allometric relationship was found in most of the chromosomal groups (see Results), subsequent analyses were conducted with the covariance matrices obtained from raw data but also from the regression residuals, in order to assess the role of allometry as an integrating factor [27]. However, and unless the converse is indicated, only the results obtained from size-corrected data are shown.

Evaluations of the hypothesis of bimodular organization of the mandible were conducted for the two components of shape, using MorphoJ. The set of digitized landmarks was subdivided into two subsets of eight and nine landmarks respectively, corresponding to the two mandibular modules (alveolar region and ascending ramus; Figure 3A). The magnitude of integration between the two subsets was quantified in each group through the computation of the $R V$ coefficient [55]. In order to assess the hypothesis of modularity, the resulting $R V$ coefficients were compared with the distributions of $R V$ coefficients obtained from alternative subsets of landmarks. These subsets were required to include the same number of landmarks as the tested subsets matching the mandibular modules. Since integration cannot occur between spatially separate units [56], comparisons were restricted to subsets whose landmarks were contiguous, that is, connected by the edges of the adjacency graph (Figure 3B). By definition, subsets of landmarks resulting from a subdivision consistent with an actual modular organization are expected to show weaker covariation, and thus lower integration, than subsets not corresponding with actual modules [4,27]. Accordingly, when the $R V$ coefficient for the two tested subsets of landmarks was lower than $95 \%$ of the distributional values, it was considered to be statistically significant $(P<0.05)$ and the hypothesis of modularity was confirmed [27]. However, it has recently been shown that 
the $R V$ coefficient decreases when sample size increases $[57,58]$. Therefore, a rarefaction procedure was used in order to obtain sample-size-corrected $R V$ values. Through a sampling with replacement, 1,000 random samples of 77 observations (sample size of the smallest group, 40Rb) were drawn from each group. Then, the $R V$ coefficient was computed for each dataset, and finally a mean $R V$ value was computed for each group [57], which was the actual sample-size-standardized $R V$ coefficient. In order to assess the dependence of the magnitude of morphological integration on allometry, a linear regression of the $R V$ coefficients from raw data on the percentages of allometry of each group was conducted. Given the low dependence of the asymmetric component of shape variation on size (see Results), this analysis was only performed for the symmetric component. Mantel and partial Mantel tests were then conducted with the aim of assessing the relationship between differences in the magnitude of morphological integration ( $R V$ coefficients) and both the karyotypic and the geographic distances between chromosomal groups (see Additional file 4). These distances were calculated following the procedure used in a previous study [28]. Both raw and size-corrected data, as well as data of the symmetric and asymmetric components, were used.

PC analyses were carried out for the symmetric and asymmetric components of shape in order to extract the patterns of variation across hemimandibles [59]. Shape changes associated with each of the first two PCs (PC1 and PC2) were visualized as diagrams of simultaneous displacements of landmarks [52]. In order to quantify the degree to which these patterns differed, vector angles between normalized PCs were calculated as the arccosine of their vector correlation (for details, see $[60,61]$ ). Calculations were conducted independently for PC1 and PC2. For each component of shape, vector angles were calculated between all pairs of groups. Additionally, the angles between corresponding PCs of the two components of shape were calculated in each group. Tests against the null hypothesis of vectors having random directions in the shape tangent space were performed through the "Compare Vector Directions" function on MorphoJ.

Similarity in the patterns of morphological integration was tested computing matrix correlations between covariance matrices. The six groups were compared in pairs for each component of shape variation separately. Besides, the correlation between the covariance matrices of the symmetric and asymmetric components was calculated in each group [34]. Matrix correlations excluded the diagonal blocks of the covariance matrices [62]. Statistical significances were determined through matrix permutation tests, with 10,000 iterations, against the null hypothesis of complete dissimilarity between the covariance matrices concerned [63]. Mantel and partial Mantel tests were conducted with the aim of assessing the possible association between differences in the patterns of morphological integration and both karyotypic and geographic distances between groups (see Additional file 4). Differences in the patterns of morphological integration between groups were calculated as 1-r, with $r$ being the correlation coefficient between the covariance matrices of the pair of groups under comparison. Both raw and size-corrected data, as well as data of the symmetric and asymmetric components, were used.

In multiple comparisons, $P$-values were adjusted by implementing the false discovery rate (FDR) procedure [64].

\section{Availability of supporting data}

The data set supporting the results of this article is provided as an additional file (Additional file 3).

\section{Additional files}

\section{Additional file 1: Two-way ANOVA of centroid size and Procrustes} ANOVA of shape for the study sample.

Additional file 2: Collection sites and individual karyotypes of the study sample, indicating the set of Rb translocations and their structural heterozygosity.

Additional file 3: Raw coordinates of the 17 landmarks digitized in the sample analyzed.

Additional file 4: Karyotypic distances (below the diagonal) and geographic distances (above the diagonal) between chromosomal groups.

\section{Abbreviations}

ANOVA: Analysis of variance; FDR: False discovery rate; F1: Filial 1; F2: Filial 2; PC: Principal component; QTLs: Quantitative trait loci; Rb: Robertsonian; St: Standard.

\section{Competing interests}

The authors declare that they have no competing interests.

\section{Authors' contributions}

JMV carried out the acquisition of morphometric data, participated in the performance of statistical analyses and drafted the manuscript. FMM conceived the study, participated in its coordination and in the performance of statistical analyses, helped to draft the manuscript and revised it critically. NM carried out the collection of the sample and the acquisition of chromosome preparations and karyotypic data. MJLF and JV revised the manuscript and gave final approval of the version to be published. JV coordinated the research project in which this study is included. All authors participated in the design of the study and the interpretation of data, and read and approved the final manuscript.

\section{Acknowledgments}

We are indebted to Christopher Evans (Servei d'Assessorament Lingüístic, University of Barcelona) for revising the English and to Dr. John Abramyan (University of British Columbia) for writing assistance. We also thank two anonymous reviewers for their valuable comments on previous drafts of this manuscript. This work was conducted in the framework of the doctoral program in Biodiversity from Universitat Autònoma de Barcelona, and was funded by the Spanish Ministerio de Economía y Competitividad (project reference CGL201015243) and by a PIF grant from Universitat Autònoma de Barcelona to JMV.

\section{Author details}

'Departament de Biologia Animal, de Biologia Vegetal i d'Ecologia, Universitat Autònoma de Barcelona, Facultat de Biociències, Bellaterra, E-08193 Cerdanyola del Vallès, Spain. ${ }^{2}$ Departament de Biologia Animal and Institut de Recerca de la Biodiversitat (IRBio), Universitat de Barcelona, Facultat de Biologia, Av. Diagonal 645, E-08028 Barcelona, Spain. 
Received: 21 March 2014 Accepted: 7 July 2014

Published: 15 July 2014

\section{References}

1. Olson EC, Miller RL: Morphological integration. Chicago: University of Chicago Press; 1958.

2. Schlosser G, Wagner GP: Modularity in development and evolution. Chicago: University of Chicago Press; 2004.

3. Klingenberg CP: Evolution and development of shape: integrating quantitative approaches. Nat Rev Genet 2010, 11:623-635.

4. Klingenberg CP: Morphological integration and developmental modularity. Annu Rev Ecol Evol Syst 2008, 39:115-132

5. Piálek J, Hauffe HC, Searle JB: Chromosomal variation in the house mouse. Biol J Linn Soc 2005, 84:535-563.

6. Bidau CJ: Causes of chiasma repatterning due to centric fusions. Braz J Genet 1993, 16:283-296.

7. Bidau CJ, Giménez MD, Palmer CL, Searle JB: The effects of Robertsonian fusions on chiasma frequency and distribution in the house mouse (Mus musculus domesticus) from a hybrid zone in northern Scotland. Heredity 2001, 87:305-313.

8. Castiglia R, Capanna E: Chiasma repatterning across a chromosomal hybrid zone between chromosomal races of Mus musculus domesticus. Genetica 2002, 114:35-40.

9. Dumas D, Britton-Davidian J: Chromosomal rearrangements and evolution of recombination: comparison of chiasma distribution patterns in standard and Robertsonian populations of the house mouse. Genetics 2002, 162:1355-1366.

10. Capilla L, Medarde N, Alemany-Schmidt A, Oliver-Bonet M, Ventura J, Ruiz-Herrera A: Genetic recombination variation in wild Robertsonian mice: on the role of chromosomal fusions and Prdm9 allelic background. Proc Biol Sci 2014. doi:10.1098/rspb.2014.0297.

11. Navarro A, Barton NH: Chromosomal speciation and molecular divergence - accelerated evolution in rearranged chromosomes. Science 2003, 300:321-324.

12. Wagner GP, Pavlicev M, Cheverud JM: The road to modularity. Nat Rev Genet 2007, 8:921-931.

13. Graham JH: Genomic coadaptation and developmental stability in hybrid zones. Acta Zool Fennica 1992, 191:121-131.

14. Grant PR, Grant BR: Phenotypic and genetic effects of hybridization in Darwin's finches. Evolution 1994, 48:297-316.

15. Wagner GP, Zhang J: The pleiotropic structure of the genotypephenotype map: the evolvability of complex organisms. Nat Rev Genet 2011, 12:204-213.

16. Schwarz E, Schwarz HK: The wild and commensal stocks of the house mouse, Mus musculus Linnaeus. J Mammal 1943, 24:59-72.

17. Adolph S, Klein J: Robertsonian variation in Mus musculus from Central Europe, Spain, and Scotland. J Hered 1981, 72:219-221.

18. Gündüz I, López-Fuster MJ, Ventura J, Searle JB: Clinal analysis of a chromosomal hybrid zone in the house mouse. Genet Res 2001, 77:41-51.

19. Sans-Fuentes MA, Muñoz-Muñoz F, Ventura J, López-Fuster MJ: Rb(7.17), a rare Robertsonian fusion in wild populations of the house mouse. Genet Res 2007, 89:207-213.

20. Medarde N, López-Fuster MJ, Muñoz-Muñoz F, Ventura J: Spatio-temporal variation in the structure of a chromosomal polymorphism zone in the house mouse. Heredity 2012, 109:78-89.

21. Atchley WR, Hall BK: A model for development and evolution of complex morphological structures. Biol Rev Camb Philos Soc 1991, 66:101-157.

22. Klingenberg CP, Navarro N: Development of the mouse mandible: a model system for complex morphological structures. In Evolution of the house mouse. Edited by Macholán M, Baird SJE, Munclinger P, Piálek J. Cambridge: Cambridge University Press; 2012:135-149.

23. Ehrich TH, Vaughn TT, Koreishi SF, Linsey RB, Pletscher LS, Cheverud JM: Pleiotropic effects on mandibular morphology I. Developmental morphological integration and differential dominance. J Exp Zool B Mol Dev Evol 2003, 296:58-79.

24. Klingenberg $C P$, Leamy $L J$, Cheverud JM: Integration and modularity of quantitative trait locus effects on geometric shape in the mouse mandible. Genetics 2004, 166:1909-1921.

25. Burgio G, Baylac M, Heyer E, Montagutelli X: Exploration of the genetic organization of morphological modularity on the mouse mandible using a set of interspecific recombinant congenic strains between $\mathrm{C} 57 \mathrm{BL} / 6$ and mice of the Mus spretus species. G3 (Bethesda) 2012, 2:1257-1268.

26. Hall BK: Unlocking the black box between genotype and phenotype: cell condensations as morphogenetic (modular) units. Biol Philos 2003, 18:219-247.

27. Klingenberg CP: Morphometric integration and modularity in configurations of landmarks: tools for evaluating a priori hypotheses. Evol Dev 2009, 11:405-421.

28. Muñoz-Muñoz F, Sans-Fuentes MA, López-Fuster MJ, Ventura J: Evolutionary modularity of the mouse mandible: dissecting the effect of chromosomal reorganizations and isolation by distance in a Robertsonian system of Mus musculus domesticus. J Evol Bio/ 2011, 24:1763-1776.

29. Jojić V, Blagojević J, Ivanović A, Bugarski-Stanojević V, Vujošević M: Morphological integration of the mandible in yellow-necked field mice: the effects of B chromosomes. J Mammal 2007, 88:689-695.

30. Jojić $\mathrm{V}$, Blagojević J, Vujošević M: B chromosomes and cranial variability in yellow-necked field mice (Apodemus flavicollis). J Mammal 2011, 92:396-406.

31. Marroig G, Cheverud JM: A comparison of phenotypic variation and covariation patterns and the role of phylogeny, ecology, and ontogeny during cranial evolution of New World monkeys. Evolution 2001, 55:2576-2600.

32. Merilä J, Björklund M: Phenotypic integration as a constraint and adaptation. In Phenotypic integration: studying the ecology and evolution of complex phenotypes. Edited by Pigliucci M, Preston K. New York: Oxford University Press; 2004:107-129.

33. Pigliucci M: Is evolvability evolvable? Nat Rev Genet 2008, 9:75-82.

34. Klingenberg CP, Mebus K, Auffray J-C: Developmental integration in a complex morphological structure: how distinct are the modules in the mouse mandible? Evol Dev 2003, 5:522-531.

35. Renaud S, Alibert P, Auffray J-C: Modularity as a source of new morphological variation in the mandible of hybrid mice. BMC Evol Biol 2012, 12:141-156.

36. Cheverud JM, Rutledge JJ, Atchley WR: Quantitative genetics of development: genetic correlations among age-specific trait values and the evolution of ontogeny. Evolution 1983, 37:895-905.

37. Wagner GP: On the eigenvalue distribution of genetic and phenotypic dispersion matrices: evidence for a nonrandom organization of quantitative character variation. J Math Biol 1984, 21:77-95.

38. Leamy L: Morphological integration of fluctuating asymmetry in the mouse mandible. Genetica 1993, 89:139-153.

39. Cheverud JM, Routman EJ, Irschick DJ: Pleiotropic effects of individual gene loci on mandibular morphology. Evolution 1997, 51:2006-2016.

40. Leamy LJ, Klingenberg CP, Sherratt E, Wolf JB, Cheverud JM: A search for quantitative trait loci exhibiting imprinting effects on mouse mandible size and shape. Heredity 2008, 101:518-526.

41. González-José R, Van der Molen S, González-Pérez E, Hernández M: Patterns of phenotypic covariation and correlation in modern humans as viewed from morphological integration. Am J Phys Anthropol 2004, 123:69-77.

42. Debat V, Alibert P, David P, Paradis E, Auffray J-C: Independence between developmental stability and canalization in the skull of the house mouse. Proc Biol Sci 2000, 267:423-430.

43. Ackermann RR: Patterns of covariation in the hominoid craniofacial skeleton: implications for paleoanthropological models. J Hum Evol 2002, 43:167-187.

44. Drake AG, Klingenberg CP: Large-scale diversification of skull shape in domestic dogs: disparity and modularity. Am Nat 2010, 175:289-301.

45. Ford CE: The use of chromosome markers. In Tissue Grafting and Radiation. Edited by Micklem HS, Loutit JF. New York: Academic; 1966:197-206.

46. Mandahl N: Methods in solid tumor cytogenetics. In Human cytogenetics. A practical approach. Edited by Rooney DE, Czepulkowski BH. London: IRL Press; 1992:155-187.

47. Klingenberg CP: MorphoJ: an integrated software package for geometric morphometrics. Mol Ecol Resour 2011, 11:353-357.

48. Dryden IL, Mardia KV: Statistical shape analysis. Chichester: Wiley; 1998.

49. Klingenberg CP, Mclntyre GS, Zaklan SD: Left-right asymmetry of fly wings and the evolution of body axes. Proc Biol Sci 1998, 265:1255-1259.

50. Klingenberg CP, Mclntyre GS: Geometric morphometrics of developmental instability: analyzing patterns of fluctuating asymmetry with Procrustes methods. Evolution 1998, 52:1363-1375.

51. Klingenberg $C P$, Barluenga $M$, Meyer $A$ : Shape analysis of symmetric structures: quantifying variation among individuals and asymmetry. Evolution 2002, 56:1909-1920.

52. Klingenberg CP, Zaklan SD: Morphological integration between developmental compartments in the Drosophila wing. Evolution 2000, 54:1273-1285 
53. Monteiro LR: Multivariate regression models and geometric morphometrics: the search for causal factors in the analysis of shape. Syst Biol 1999, 48:192-199.

54. Good P: Permutation tests: a practical guide to resampling methods for testing hypotheses. New York: Springer; 1994.

55. Escoufier Y: Le traitement des variables vectorielles. Biometrics 1973 29:751-760.

56. Martínez-Abadías N, Esparza M, Sjøvold T, González-José R, Santos M, Hernández M, Klingenberg CP: Pervasive genetic integration directs the evolution of human skull shape. Evolution 2012, 66:1010-1023.

57. Fruciano C, Franchini P, Meyer A: Resampling-based approaches to study variation in morphological modularity. PLoS One 2013, 8:e69376. doi:10.1371/journal.pone.0069376.

58. Smilde AK, Kiers HAL, Bijlsma S, Rubingh CM, van Erk MJ: Matrix correlations for high-dimensional data: the modified RV-coefficient. Bioinformatics 2009, 25:401-405.

59. Jolliffe IT: Principal component analysis. New York: Springer; 1986.

60. Klingenberg CP: Multivariate allometry. In Advances in morphometrics. Edited by Marcus LF, Corti M, Loy A, Naylor GJP, Slice DE. New York: Plenum Press; 1996:23-49.

61. Young RL, Badyaev AV: Evolutionary persistence of phenotypic integration: influence of developmental and functional relationships on complex trait evolution. Evolution 2006, 60:1291-1299.

62. Klingenberg CP, Badyaev AV, Sowry SM, Beckwith NJ: Inferring developmental modularity from morphological integration: analysis of individual variation and asymmetry in bumblebee wings. Am Nat 2001, 157:11-23.

63. Cheverud JM, Wagner GP, Dow MM: Methods for the comparative analysis of variation patterns. Syst Zool 1989, 38:201-213.

64. Benjamini $Y$, Hochberg $Y$ : Controlling the false discovery rate: a practical and powerful approach to multiple testing. J R Stat Soc Series B Stat Methodol 1995, 57:289-300.

doi:10.1186/s12983-014-0051-3

Cite this article as: Martínez-Vargas et al:: Effect of chromosomal reorganizations on morphological covariation of the mouse mandible: insights from a Robertsonian system of Mus musculus domesticus. Frontiers in Zoology 2014 11:51.

\section{Submit your next manuscript to BioMed Central and take full advantage of:}

- Convenient online submission

- Thorough peer review

- No space constraints or color figure charges

- Immediate publication on acceptance

- Inclusion in PubMed, CAS, Scopus and Google Scholar

- Research which is freely available for redistribution 